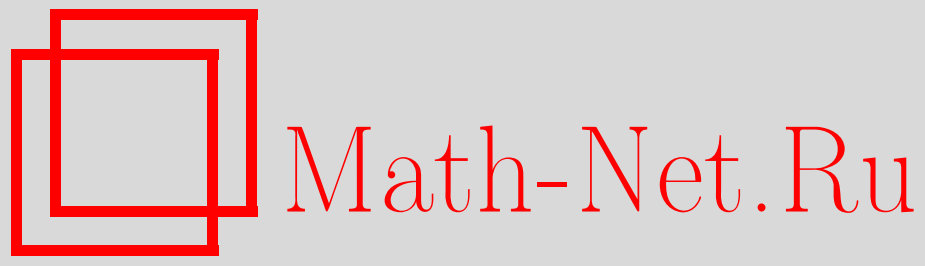

А. Ю. Константинов, О существенном спектре одного класса матричных дифференциальных операторов, Функи. анализ и его прил., 2002, том 36, выпуск $3,76-78$

DOI: https://doi.org/10.4213/faa209

Использование Общероссийского математического портала Math-Net.Ru подразумевает, что вы прочитали и согласны с пользовательским соглашением

http://www.mathnet.ru/rus/agreement

Параметры загрузки:

IP : 54.197 .130 .99

26 апреля 2023 г., 13:40:44

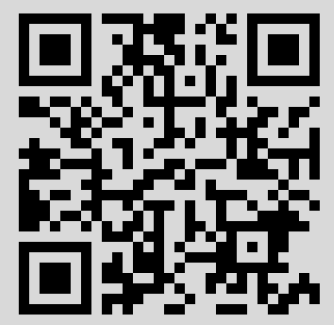


УДК 517.9

\title{
О существенном спектре одного класса матричных дифференциальных операторов
}

\author{
(c) 2002.А.Ю.КонСтантинов
}

Задача о существенном спектре матричных дифференциальных операторов смешанного порядка изучалась многими авторами. Наиболее общие результаты для эллиптических по Дуглису-Ниренбергу систем были получены в $[1]$. В $[2,3]$ (см. также [4]) была предложена абстрактная схема для исследования существенного спектра блочных операторных матриц. Отметим также статьи [5-7], где эта схема применялась к матричным дифференциальным операторам. В частности, в $[5,8]$ был вычислен существенный спектр матричного дифференциального оператора смешанного порядка, связанного с моделью линейных колебаний идеально проводящей невязкой плазмы во внешнем прямом магнитном поле (см. [9]). В настоящей работе мы развиваем результаты работ $[5,8]$ и исследуем класс неэллиптических дифференциальных операторов вида

$$
L=\left(\begin{array}{ccc}
-\rho^{-1} \partial_{1} \rho a \partial_{1}+b_{11} & -\rho^{-1} \partial_{1} \rho a \partial_{2}+b_{12} & -\rho^{-1} i \partial_{1} \rho c_{1} \\
-\rho^{-1} \partial_{2} \rho a \partial_{1}+b_{21} & -\rho^{-1} \partial_{2} \rho a \partial_{2}+b_{22} & -\rho^{-1} i \partial_{2} \rho c_{1} \\
-i c_{2} \partial_{1} & -i c_{2} \partial_{2} & d
\end{array}\right),
$$

$D(L)=\left\{f=\left(f_{1}, f_{2}, f_{3}\right) \in\left(W_{2}^{1}(\Omega)\right)^{3}\left|\partial_{1} f_{1}+\partial_{2} f_{2} \in W_{2}^{1}(\Omega), \nu_{1} f_{1}+\nu_{2} f_{2}\right|_{\partial \Omega}=0\right\}$,

в пространстве $\mathscr{H}=\left(L_{2}(\Omega, \rho)\right)^{3}$. Здесь $\Omega \subset \mathbb{R}^{2}-$ ограниченная область с границей класса $C^{2}$ (условия гладкости на границу могут быть ослаблены (см. [5])), $L_{2}(\Omega, \rho)$ - пространство квадратично суммируемых функций на области $\Omega$ с весом $\rho$, в $\mathscr{H}$ вводится метрика ортогональной суммы гильбертовых пространств, $\partial_{1}=\partial / \partial x_{1}, \partial_{2}=\partial / \partial x_{2}$ и $\nu=\left(\nu_{1}, \nu_{2}\right)$ - вектор внешней нормали к $\partial \Omega$. Предполагается, что функции $a, c_{1}, c_{2}, \rho$ удовлетворяют условию Липшица на $\bar{\Omega}$, $b_{i j}, d \in C(\bar{\Omega})$. Более того, $а$ и $\rho$ положительны на $\bar{\Omega}\left(b_{i j}, c_{1}, c_{2}, d-\right.$ комплекснозначные функции на $\bar{\Omega})$.

Оператор $L$ удобно записать в виде блочной операторной матрицы (ниже $B:=$ $\left(b_{i j}\right)_{i, j=1}^{2}-$ матричный потенциал):

$$
L=\left(\begin{array}{ll}
L_{11} & L_{12} \\
L_{21} & L_{22}
\end{array}\right)=\left(\begin{array}{cc}
-\rho^{-1} \nabla \rho a \operatorname{div}+B & -i \rho^{-1} \nabla \rho c_{1} \\
-i c_{2} \operatorname{div} & d
\end{array}\right)
$$

в ортогональной сумме $\mathscr{H}=\mathscr{H}_{1} \oplus \mathscr{H}_{2}=\left(L_{2}(\Omega, \rho)\right)^{2} \oplus L_{2}(\Omega, \rho)$.

Напомним, что замкнутый оператор $S$ называется фредгольмовым, если его ядро $\operatorname{Ker}(S)$ конечномерно и образ $\operatorname{Ran}(S)$ - замкнутое подпространство конечной коразмерности. Существенный спектр $\sigma_{\text {ess }}(S)$ замкнутого оператора $S$ определяется как множество тех $\lambda \in \mathbb{C}$, для которых оператор $S-\lambda$ не фредгольмов.

Остановимся сначала на анализе оператора $L_{11}=A+B$, где $A=-\rho^{-1} \nabla \rho a \operatorname{div}$, $D(A)=D\left(L_{11}\right)=\left\{f=\left(f_{1}, f_{2}\right) \in\left(W_{2}^{1}(\Omega)\right)^{2} \mid \partial_{1} f_{1}+\partial_{2} f_{2} \in W_{2}^{1}(\Omega), \nu_{1} f_{1}+\right.$ $\left.\left.\nu_{2} f_{2}\right|_{\partial \Omega}=0\right\}$. Оператор $A$ неотрицателен в пространстве $\mathscr{H}_{1}$, более того, он существенно самосопряжен [5] и его замыкание может быть представлено в виде произведения $\bar{A}=T T^{*}$, где $T=-\rho^{-1} \nabla a^{1 / 2}$ ( $T$ рассматривается как оператор из 
$L_{2}\left(\Omega, \rho^{-1}\right)$ в $\mathscr{H}_{1}$ на области $\left.D(T)=W_{2}^{1}(\Omega)\right)$. При этом сопряженный оператор $T^{*}$ действует из $\mathscr{H}_{1}$ в $L_{2}\left(\Omega, \rho^{-1}\right)$ на соответствующей области определения. Так как сужения операторов $T T^{*}$ и $T^{*} T$ на ортогональные дополнения к их ядрам унитарно эквивалентны (см., например, [10]), то отсюда легко вытекает, что спектр оператора $\bar{A}$ состоит из нуля (собственное значение бесконечной кратности) и последовательности положительных собственных значений конечной кратности, стремящейся к $\infty$ (в простейшей ситуации, при $\rho=a=1, T^{*} T-$ просто лапласиан с граничными условиями Неймана). В частности, $\sigma_{\mathrm{ess}}(\bar{A})=\{0\}$.

Применяя классическую теорему Вейля (см. [11]) о сохранении существенного спектра при относительно компактных возмущениях, можно показать (см. [5]), что

$$
\sigma_{\mathrm{ess}}(\bar{A}+B)=\sigma_{\mathrm{ess}}(\widehat{B}) .
$$

Здесь $\widehat{B}-$ ограниченный оператор в $\mathscr{K}=\operatorname{Ker}(\bar{A})$, задаваемый соотношением $\widehat{B} f=P B f$, где $P-$ ортопроектор в $\mathscr{H}_{1}$ на подпространство $\mathscr{K}$. Аналогично, для фиксированного $x \in \bar{\Omega}$ введем оператор $\widehat{B}(x) f=P B(x) f, f \in \mathscr{K}$. Нам понадобится также симметризованная матрица $B_{s}(x):=\left(\tilde{b}_{i j}(x)\right)_{i, j=1}^{2}, \tilde{b}_{i j}(x)=$ $\left(b_{i j}(x)+b_{j i}(x)\right) / 2$. Ниже $f(\bar{\Omega})=\{f(x) \mid x \in \bar{\Omega}\}$, а $[\lambda, \mu]$ - отрезок, соединяющий точки $\lambda, \mu \in \mathbb{C}$.

Теорема 1. Предположим, ито коэффициенты оператора $L_{11}$ удовлетворяют перечисленным выше условиям. Тогда

$$
\sigma_{\mathrm{ess}}\left(\overline{L_{11}}\right)=\bigcup_{x \in \bar{\Omega}} \sigma_{\mathrm{ess}}(\widehat{B}(x))=\bigcup_{x \in \bar{\Omega}} \sigma_{\mathrm{ess}}\left(\widehat{B}_{s}(x)\right) .
$$

Если дополнительно предположить, ито симметризованная матрица $B_{s}(x)$ вещественна для всех $x \in \Omega$, то

$$
\sigma_{\mathrm{ess}}\left(\bar{L}_{11}\right)=[m, M] .
$$

Здесь $m, M-$ соответственно нижняя и верхняя грани оператора умножения на матричнозначную функцию $B_{s}$ в $\mathscr{H}_{1}$. Если же $B_{s}$ диагональна, то

$$
\sigma_{\text {ess }}\left(\bar{L}_{11}\right)=\bigcup_{x \in \bar{\Omega}}\left[b_{11}(x), b_{22}(x)\right] .
$$

Отметим, что если $B:=\left(b_{i j}\right)_{i, j=1}^{2}=b I$, где $I-$ единичная матрица в $\mathbb{C}^{2}$, результат теоремы 1 легко вытекает из $(3)$ и компактности коммутатора $[P, \varphi]$, где $\varphi-$ оператор умножения на функцию $\varphi \in C(\bar{\Omega})$. В этом случае (см. [5]) $\sigma_{\text {ess }}\left(\bar{L}_{11}\right)=\sigma_{\text {ess }}(\widehat{B})=b(\bar{\Omega})$.

Для матрицы $B$ общего вида задача вычисления существенного спектра оператора $\widehat{B}$ значительно сложнее. Поясним результат в случае постоянных $B$ и $\rho$. Более того, дополнительно предположим, что матрица $B$ диагональна, а $\Omega-$ прямоугольник со сторонами, параллельными осям координат. Заметим, что уравнение $\widehat{B} f=\lambda f$ эквивалентно соотношению

$$
(B-\lambda) f=\nabla g, \quad f \in \mathscr{K},
$$

где $g \in W_{2}^{1}(\Omega)$. Последнее вытекает из того, что ортогональное дополнение к $\mathscr{K}$ состоит из потенциальных полей (см., например, [12]), т. е. $\mathscr{K}^{\perp}=\{\nabla g \mid g \in$ 
$\left.W_{2}^{1}(\Omega)\right\}$. Отсюда (по крайней мере для гладких $g$ ) следует, что $g-$ решение краевой задачи

$$
\left(b_{22}-\lambda\right) \partial_{11}^{2} g+\left(b_{11}-\lambda\right) \partial_{22}^{2} g=0, \quad \nu_{1} \partial_{1} g+\left.\frac{b_{11}-\lambda}{b_{22}-\lambda} \nu_{2} \partial_{2} g\right|_{\partial \Omega}=0 .
$$

Наоборот, при $\lambda \notin\left\{b_{11}, b_{22}\right\}$ всякое (отличное от постоянной) решение задачи (6) восстанавливает по (5) собственную функцию оператора $\widehat{B}$. Явно решая (6), нетрудно показать, что оператор $\widehat{B}$ имеет полную систему ортонормированных собственных векторов и его собственные числа всюду плотны в отрезке $\left[b_{11}, b_{22}\right]$, что и доказывает (4) в рассмотренной здесь модельной ситуации.

Теорема 2. Пусть функциии $a, c_{1}, c_{2}, \rho$ удовлетворяют условию Липшица на $\bar{\Omega}$, а и $\rho$ положительны на $\bar{\Omega}, b_{i j}, d \in C(\bar{\Omega})$. Тогда оператор $L$ допускает замыкание и

$$
\sigma_{\mathrm{ess}}(\bar{L})=\bigcup_{x \in \bar{\Omega}} \sigma_{\mathrm{ess}}(\widehat{B}(x)) \cup\left(d-\frac{c_{1} c_{2}}{a}\right)(\bar{\Omega}) .
$$

Отметим, что в случае скалярной матрицы $B=b I$ результаты теорем 1,2 установлены в [5]. В [13] был рассмотрен случай диагональной матрицы $B$.

Поясним схему доказательства теоремы 2. Оно использует теорему 1 и абстрактный подход к изучению существенного спектра операторных матриц, основанный на факторизации операторной матрицы $L-\mu$ в смысле Фробениуса-Шура (см. $[2-5])$. При этом важную роль играет компактность операторов

$$
\bar{L}_{21}\left(\bar{L}_{11}-\mu\right)^{-1}, \quad L_{12}^{*}\left(\bar{L}_{11}-\mu\right)^{-1},
$$

где $\mu-$ произвольная точка из резольвентного множества оператора $\bar{L}_{11}$. Теперь несложно показать, что

$$
\sigma_{\mathrm{ess}}(\bar{L})=\sigma_{\mathrm{ess}}\left(\bar{L}_{11}\right) \cup \sigma_{\mathrm{ess}}\left(\overline{L_{22}-L_{21}\left(\bar{L}_{11}-\mu\right)^{-1} L_{12}}\right)=\sigma_{\mathrm{ess}}\left(\bar{L}_{11}\right) \cup\left(d-\frac{c_{1} c_{2}}{a}\right)(\bar{\Omega}) .
$$

В заключение отметим, что развиваемая в данной работе техника работает и в более общих ситуациях. В частности, теорема 2 сохраняется и для многомерного варианта оператора вида $(2)$, действующего в $\left(L_{2}(\Omega, \rho)\right)^{n} \oplus L_{2}(\Omega, \rho)$. Здесь $\Omega-$ ограниченная область с достаточно гладкой границей в $\mathbb{R}^{n}$.

\section{ЛИТЕРАТУРА}

1. Grubb G., Geymonat G. Math. Ann., 227, 247-276 (1977). 2. Atkinson F. V., Langer H., Mennicken R., Shkalikov A. A. Math. Nachr., 167, 5-20 (1994). 3. Шкаликов A. А. Матем. заметки, 58, 945-949 (1995). 4. Konstantinov A. Yu. Укр. матем. ж., 50, 1064-1072 (1998). 5. Langer H., Möller M. Math. Nachr., 178, 233-248 (1996). 6. Hardt V., Mennicken R., Naboko S. Math. Nachr., 205, 19-68 (1999). 7. Константинов А. Ю. Функц. анализ и его прил., 31, вып. 3, 77-80 (1997). 8. Raikov G. D. Asymptotic Analysis, 3, 1-35 (1990). 9. Lifschitz A. E. Magnetohydrodynamics and spectral theory. Kluwer Acad. Publ., Dodrecht, 1989. 10. Deift P. A. Duke Math. J., 45, 267-310 (1978). 11. Kaто T. Теория возмущений линейных операторов. Мир, М. (1972). 12. Копачевский Н. Д., Крейн С. Г., Нго Зуй Кан. Операторные методы в линейной гидродинамике. Наука, М., 1989. 13. Гринык Ю. Н., Константинов А. Ю. Доповіді НАН України, № 7, 13-17 (2002).

Киевский университет, кафедра математического анализа e-mail: konst@faust.kiev.ua 